\title{
Autocrine IFN-I inhibits isocitrate dehydrogenase in the TCA cycle of LPS-stimulated macrophages
}

\author{
David P. De Souza, ${ }^{1}$ Adrian Achuthan, ${ }^{2}$ Man K.S. Lee, ${ }^{3}$ Katrina J. Binger, ${ }^{4}$ Ming-Chin Lee, ${ }^{2}$ Sophia Davidson, ${ }^{5}$ Dedreia L. Tull, \\ Malcolm J. McConville, ${ }^{1,4}$ Andrew D. Cook, ${ }^{2}$ Andrew J. Murphy, John A. Hamilton, ${ }^{2,6}$ and Andrew J. Fleetwood ${ }^{2}$
}

\begin{abstract}
'Metabolomics Australia, Bio21 Molecular Science and Biotechnology Institute, University of Melbourne, Parkville, Victoria, Australia. 2Department of Medicine, University of Melbourne, Royal Melbourne Hospital, Parkville, Victoria, Australia. ${ }^{3}$ Haematopoiesis and Leukocyte Biology, Baker IDI Heart and Diabetes Institute, Melbourne, Victoria, Australia. ${ }^{4}$ Department of Biochemistry and Molecular Biology, Bio21 Molecular Science and Biotechnology Institute, University of Melbourne, Parkville, Victoria, Australia. Innflammation Division, Walter and Eliza Hall Institute of Medical Research, Parkville, Victoria, Australia. ${ }^{6}$ Australian Institute for Musculoskeletal Science (AIMSS), University of Melbourne and Western Health, St. Albans, Victoria, Australia.

Macrophage activation in response to LPS is coupled to profound metabolic changes, typified by accumulation of the TCA cycle intermediates citrate, itaconate, and succinate. We have identified that endogenous type I IFN controls the cellular citrate/ $\alpha$-ketoglutarate ratio and inhibits expression and activity of isocitrate dehydrogenase (IDH); and, via ${ }^{13} \mathrm{C}$-labeling studies, demonstrated that autocrine type I IFN controls carbon flow through IDH in LPS-activated macrophages. We also found that type I IFN-driven IL-10 contributes to inhibition of IDH activity and itaconate synthesis in LPS-stimulated macrophages. Our findings have identified the autocrine type I IFN pathway as being responsible for the inhibition of IDH in LPS-stimulated macrophages.
\end{abstract}

\section{Introduction}

Macrophages activated with LPS (so-called M1 macrophages) have a TCA cycle that is altered at 2 distinct steps, leading to the accumulation of citrate, succinate, and itaconate (1). The mechanisms leading to the increased levels of these metabolites following LPS activation are not fully understood. It has been found that LPS inhibits gene expression of isocitrate dehydrogenase (IDH) $(2,3)$, the enzyme that converts isocitrate to $\alpha$-ketoglutarate and a key checkpoint in the TCA cycle. Repression of Idh mRNA leads to reduced $\alpha$-ketoglutarate levels and accumulation of citrate in activated macrophages $(2,3)$. Inhibition of Idh represents the first "break" in the TCA cycle of LPS-treated macrophages (2-4). Citrate can promote inflammatory responses in macrophages and is also a precursor for the generation of itaconate (4). The second break in the TCA cycle is associated with accumulation of succinate, which is derived from enhanced glutaminolysis (3). Oxidation of succinate by succinate dehydrogenase $(\mathrm{SDH})$ in the electron transport chain drives macrophage ROS and IL-1 $\beta$ production (5). Apart from being a powerful antibacterial agent (6), itaconate inhibits the enzymatic activity of SDH in LPS-stimulated macrophages (7).

Type I IFNs are constitutively produced in a range of tissues and cells, and are critical regulators of innate and adaptive immune response (8). LPS-stimulated macrophages are a substantial source of IFN- $\beta$, which in turn signals via its receptors (IFNAR1 and IFNAR2) to induce production of mediators such as IL-12p70, NO, and IL-10 (9). Given the recent interest in the autocrine action of cytokines (e.g., IL-10) in regulating macrophage

Conflict of interest: The authors have declared that no conflict of interest exists. Copyright: (c) 2019, American Society for Clinical Investigation.

Submitted: January 21, 2019; Accepted: July 9, 2019; Published: September 4, 2019.

Reference information: J Clin Invest. 2019;129(10):4239-4244.

https://doi.org/10.1172/JCl127597. metabolism (10), we wanted to address the role of endogenous type I IFN in regulating the profound metabolic changes observed in LPS-activated macrophages. Here we report that the type I IFN pathway is responsible for the truncated TCA cycle at IDH and accumulation of citrate in LPS-stimulated macrophages.

\section{Results and Discussion}

Autocrine type I IFN promotes TCA cycle fragmentation and citrate accumulation in LPS-treated macrophages. To investigate whether endogenous type I IFN is involved in regulating macrophage metabolism, we stimulated bone marrow-derived macrophages (BMMs) from WT or Ifnar1 ${ }^{-/-}$mice with LPS $(100 \mathrm{ng} / \mathrm{mL})$ or vehicle (PBS) for 24 hours and profiled changes in metabolite levels by gas chromatography-mass spectrometry (GC-MS). As expected, LPS treatment of WT BMMs led to accumulation of itaconate, succinate, and citrate (refs. 2, 3, and Figure 1A; see Supplemental Tables 1-4 for the full list of metabolites; supplemental material available online with this article; https://doi.org/10.1172/JCI127597DS1). LPS treatment also led to reduced levels of isocitrate and $\alpha$-ketoglutarate in WT BMMs, in agreement with an LPS-induced breakpoint in the TCA cycle at IDH (2).

Similar to others (11), we observed that IFN- $\beta$ treatment enhanced expression of Irg1 (the gene encoding the enzyme that synthesizes itaconate; ref. 6) (Supplemental Figure 1A) and that autocrine type I IFN facilitated LPS-mediated induction of Irg1 in BMMs (Supplemental Figure 1B). However, LPS treatment of Ifnar ${ }^{-/-}$BMMs did increase Irg1 gene transcription (Figure 1A and Supplemental Figure 1B), and they expressed Irg1 protein levels similar to those in WT BMMs (Figure 1A). Blockade of endogenous IFN- $\beta$ with a neutralizing Ab significantly reduced, but did not completely abrogate, Irg1 expression in LPS-stimulated BMMs (Supplemental Figure 1C) and had no observable impact on Irg1 protein levels (Supplemental Figure 1D). Irg1 gene (Supplemental 
$\mathbf{A}$
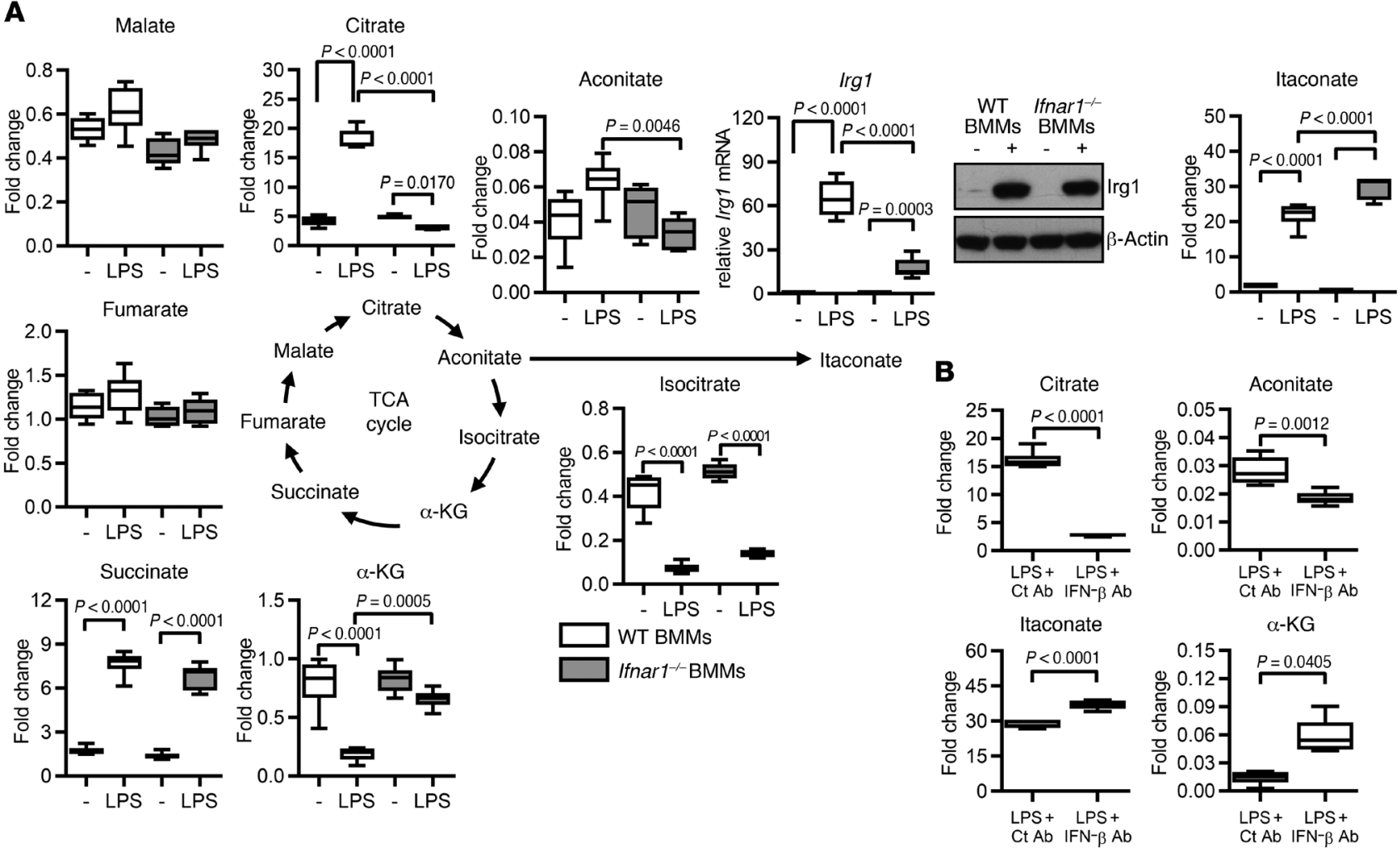

Figure 1. Autocrine type I IFN promotes TCA cycle fragmentation and citrate accumulation in LPS-treated macrophages. Metabolic profiling of WT and Ifnar ${ }^{-1-}$ BMMs stimulated or not with LPS $(100 \mathrm{ng} / \mathrm{mL})$ for 24 hours was carried out by GC-MS. (A) Schematic diagram of the TCA cycle, with the abundance of individual metabolites quantified. Data (fold change) are presented as histograms $(n=6)$ ). Gene and protein levels of Irg1 were determined by qPCR $(n=4)$ and Western blot analysis. (B) Citrate, aconitate, itaconate, and $\alpha$-ketoglutarate levels determined by GC-MS in WT BMMs stimulated with LPS (100 ng/mL, 24 hours) in the presence of $50 \mathrm{U} / \mathrm{mL}$ of an IFN- $\beta$-neutralizing Ab or isotype control lgG $(250 \mathrm{ng} / \mathrm{mL}$, corresponding to $50 \mathrm{U} / \mathrm{mL}$ IFN- $\beta$ Ab). $P$ values were determined by 1-way ANOVA (A) or Student's $t$ test (B). $\alpha$-KG, $\alpha$-ketoglutarate; $C \mathrm{C}$, control.

Figure 1E) and protein (Supplemental Figure $1 \mathrm{~F})$ expression was completely abolished in poly(I:C)-treated Ifnar1 ${ }^{-/}$BMMs. Steadystate levels of itaconate were lower in Ifnar $1^{-/-}$than in WT BMMs (Supplemental Table 4), which may reflect the basal release of IFN- $\beta$ by BMMs (9). Surprisingly, LPS-induced itaconate accumulation was greater in Ifnar1-- than in WT BMMs (Supplemental Table 1 and Figure 1A). The marked increase in the generation of itaconate in LPS-stimulated Ifnar1 ${ }^{--}$BMMs was coupled to a significant reduction in the pool sizes of the upstream precursors citrate and aconitate (Figure 1A) relative to LPS-stimulated WT BMM. Thus, despite the finding that autocrine type I IFN facilitated an increase in LPS-induced Irg1 expression (Figure 1A and Supplemental Figure 1, B and C), Ifnar1 ${ }^{-/}$BMMs were able to synthesize large pools of itaconate (Figure 1A).

WT and Ifnar1 ${ }^{-1}$ BMMs had a reduced mitochondrial oxygen consumption rate (OCR), increased extracellular acidification rate (ECAR), and pronounced lactate secretion following LPS stimulation (Supplemental Figure 1G). Similar to LPS-stimulated WT BMMs, Ifnar1 ${ }^{-/}$BMMs had expanded pools of succinate (Figure 1A) following LPS activation. However, in stark contrast to WT BMMs, Ifnar1 ${ }^{-/}$BMMs had unchanged $\alpha$-ketoglutarate levels and significantly reduced citrate levels after LPS activation ( $\sim 1.5$-fold lower in LPS-treated vs. untreated cells; Figure 1A). To avoid any potential developmental issues with Ifnar $1^{-1-}$ mice, we undertook metabolic profiling of LPS- treated WT BMMs in the presence of a neutralizing anti-IFN- $\beta$ $\mathrm{Ab}$ or an isotype control Ab. As summarized in Figure 1B and Supplemental Figure $1 \mathrm{H}$ (see Supplemental Table 5 for the full list of metabolites), neutralization of IFN- $\beta$ led to a significant reduction in the accumulation of citrate and aconitate, with a corresponding increase in the $\alpha$-ketoglutarate and itaconate pools in LPS-stimulated BMMs relative to isotype control-treated cells. These data are consistent with the changes observed in LPS-treated Ifnar1 ${ }^{-/}$BMMs (Figure 1A) and together suggest that autocrine type I IFN is responsible for the inhibition of IDH in the TCA cycle of LPS-stimulated macrophages (2).

Autocrine type I IFN controls the carbon flow through IDH in LPS-treated macrophages. Impaired carbon flow through IDH in LPS-stimulated macrophages supports the production of citrate (2). To examine whether the lack of citrate accumulation observed in the absence of endogenous type I IFN signaling (Figure 1, A and B) was due to altered carbon flow through IDH, we conducted stable isotopic labeling experiments to trace the fate of ${ }^{13} \mathrm{C}$-glucose in untreated and LPS-treated WT and Ifnar ${ }^{-/} \mathrm{BMMs}$, as well as in LPS-treated WT BMMs in the presence of anti-IFN- $\beta$ Ab or isotype control Ab (we used a 24-hour time point, with labeling initiated at the time of LPS treatment). To capture any difference in carbon flow through IDH, we measured the relative rates of glucosederived carbon accumulation in the TCA cycle upstream (i.e., citrate) and downstream (i.e., succinate) of IDH. 
A

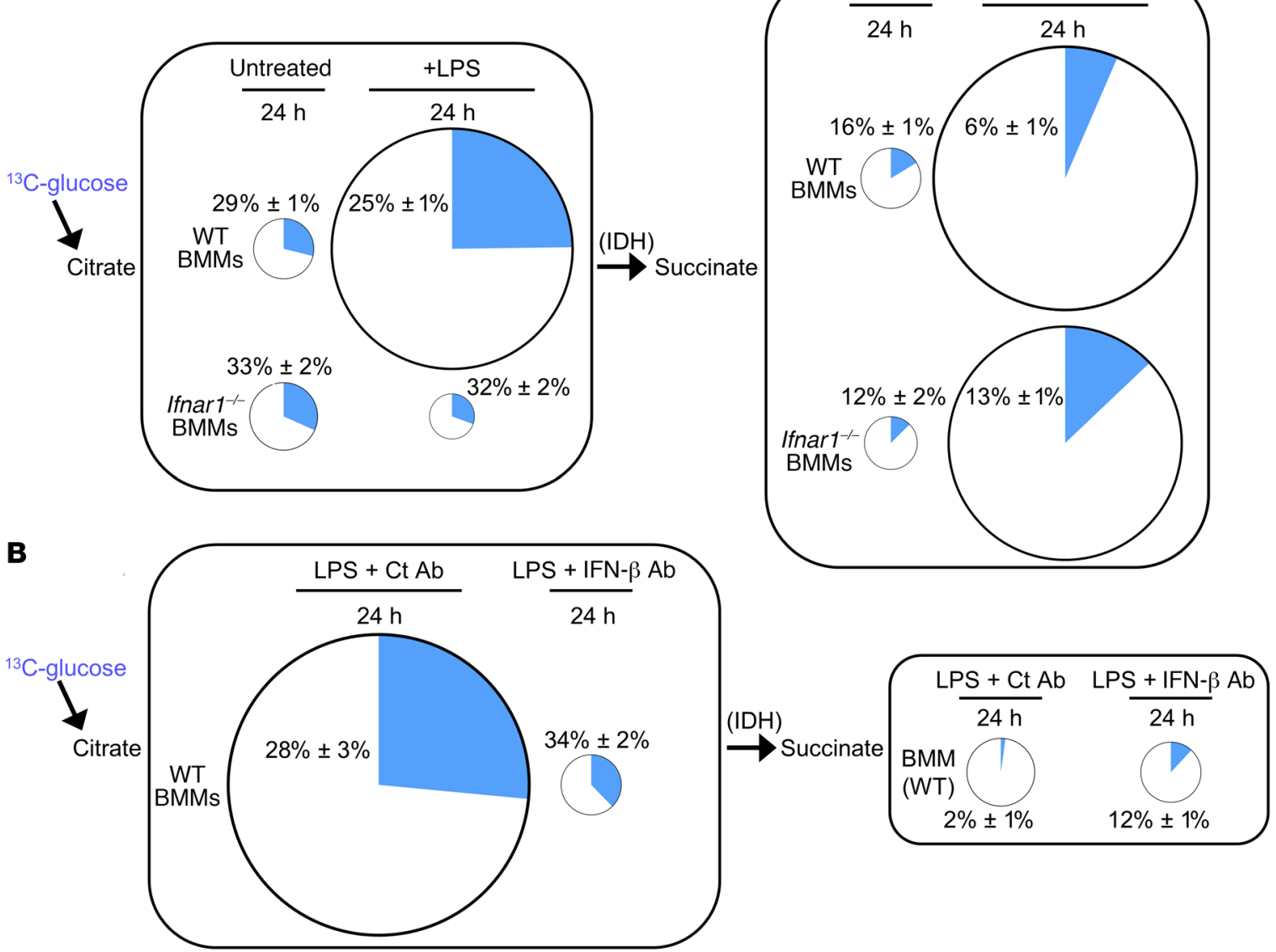

Figure 2. Autocrine type I IFN controls carbon flow through IDH in LPS-treated macrophages. ${ }^{13} \mathrm{C}$-glucose labeling of citrate and succinate in (A) BMMs (WT and Ifnar1 ${ }^{--}$) stimulated or not with LPS (100 ng/mL, 24 hours) or (B) WT BMMs stimulated with LPS (100 $\mathrm{ng} / \mathrm{mL}, 24$ hours) in the presence of IFN- $\beta$ $\mathrm{Ab}(50 \mathrm{U} / \mathrm{mL})$ or Ct Ab $\left(250 \mathrm{ng} / \mathrm{mL}\right.$, corresponding to $50 \mathrm{U} / \mathrm{mL}$ anti-IFN- $\beta$ Ab). Cells were treated with ${ }^{13} \mathrm{C}$-glucose (10 mM) for 24 hours (i.e., labeling was initiated at the time of LPS treatment). ${ }^{13} \mathrm{C}$ incorporation was analyzed by GC-MS. Mean percentage labeling \pm SEM $(n=3)$ is shown. See Supplemental Figure $1, I$ and J, for ${ }^{13} \mathrm{C}$-glucose labeling of itaconate. Circle sizes are scaled with respect to pool sizes relative to untreated WT BMMs in A, or to BMMs + LPS + Ct Ab in B, for each metabolite.

Basal ${ }^{13} \mathrm{C}$-glucose incorporation in citrate was similar in WT and Ifnar1 ${ }^{--}$BMMs. Consistent with a disrupted carbon flow through IDH in WT BMMs, we found that the proportion of glucose-derived carbon in succinate fell from approximately $16 \%$ $\pm 1 \%$ to $6 \% \pm 1 \%$ following LPS stimulation (Figure $2 \mathrm{~A}$ ). In comparison, approximately $12 \% \pm 2 \%$ of succinate was synthesized from glucose in untreated Ifnar1 ${ }^{-/}$BMMs, and following LPS stimulation, this was maintained at $13 \% \pm 1 \%$, suggesting that carbon flow from glucose, via IDH, to succinate is uninterrupted in the absence of endogenous type I IFN in LPS-activated BMMs. An unperturbed carbon flow through IDH in LPS-stimulated Ifnar ${ }^{-/-}$BMMs is in line with the reduced citrate pool size and the maintained $\alpha$-ketoglutarate levels in these cells (Figure 1A). The LPS-induced reduction of citrate in LPS-stimulated Ifnar1 ${ }^{-1-}$ BMMs is also likely due to it being utilized to sustain the large accumulation of LPS-induced itaconate in these cells ( 100 -fold vs. untreated cells; Figure 1A and Supplemental Table 3). Consistent with this, we found significantly increased ${ }^{13} \mathrm{C}$-glucose incorporation into itaconate pools in LPS-stimulated versus untreated Ifnar ${ }^{-1-}$ BMMs (Supplemental Figure 1I), which was not evident in WT BMMs (Supplemental Figure 1I).
Blockade of endogenous type I IFN with a neutralizing antiIFN $-\beta \mathrm{Ab}$ increased carbon flow through IDH in LPS-stimulated BMMs. ${ }^{13} \mathrm{C}$-glucose incorporation in succinate increased from approximately $2 \% \pm 1 \%$ in LPS plus control Ab-treated BMMs to $12 \% \pm 1 \%$ in LPS plus anti-IFN- $\beta$ Ab-treated BMMs (Figure $2 \mathrm{~B})$. The restored carbon flow through IDH in LPS + anti-IFN- $\beta$ $\mathrm{Ab}$-treated BMMs is consistent with the reduced citrate and aconitate as well as the elevated $\alpha$-ketoglutarate pools in these cells (Figure 1B). In line with our findings in Ifnar1 ${ }^{--}$BMMs (Supplemental Figure 1I), we found that LPS plus anti-IFN- $\beta$ Ab-treated WT BMMs had enhanced flux toward itaconate (Supplemental Figure 1J). These findings suggest that the elevated itaconate pools observed in the absence of endogenous type I IFN in LPS-activated BMMs (Figure 1, A and B) are supported by increased flux of glucose-derived carbon toward itaconate. Our data indicate that autocrine type I IFN controls the carbon flow through IDH in LPS-stimulated macrophages.

Autocrine type I IFN inhibits IDH in LPS-treated macrophages. Five genes (Idh1, Idh2, Idh $3 a, I d h 3 b$, and Idh $3 g$ ) encode for 3 IDH catalytic isozymes: IDH1, IDH2, and IDH3 (12). We found that LPS-stimulated WT BMMs had significantly decreased expres- 
A
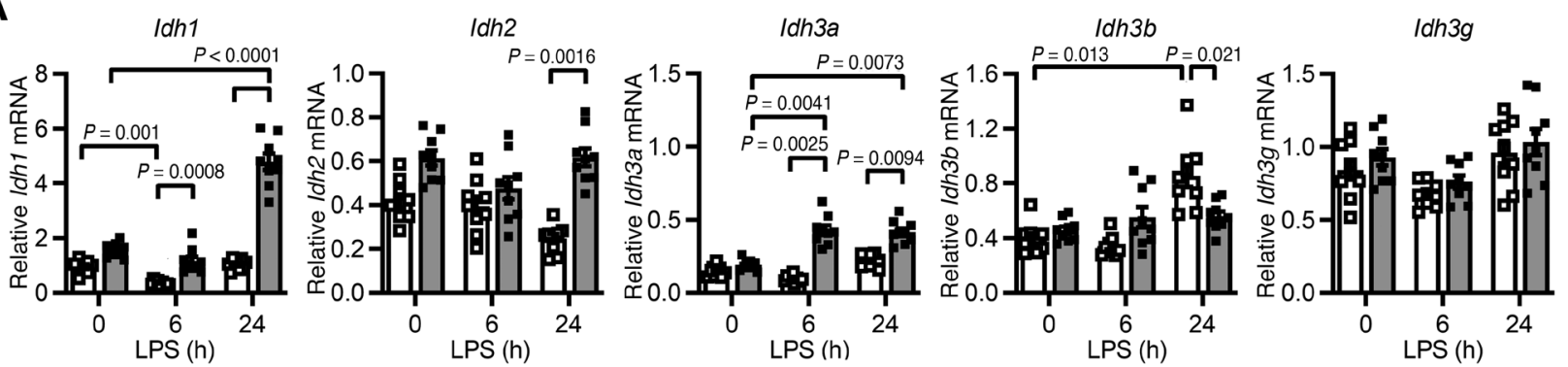

B

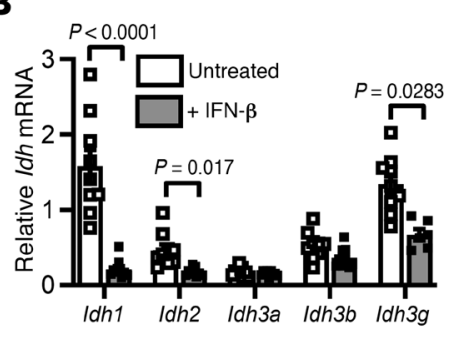

C

D
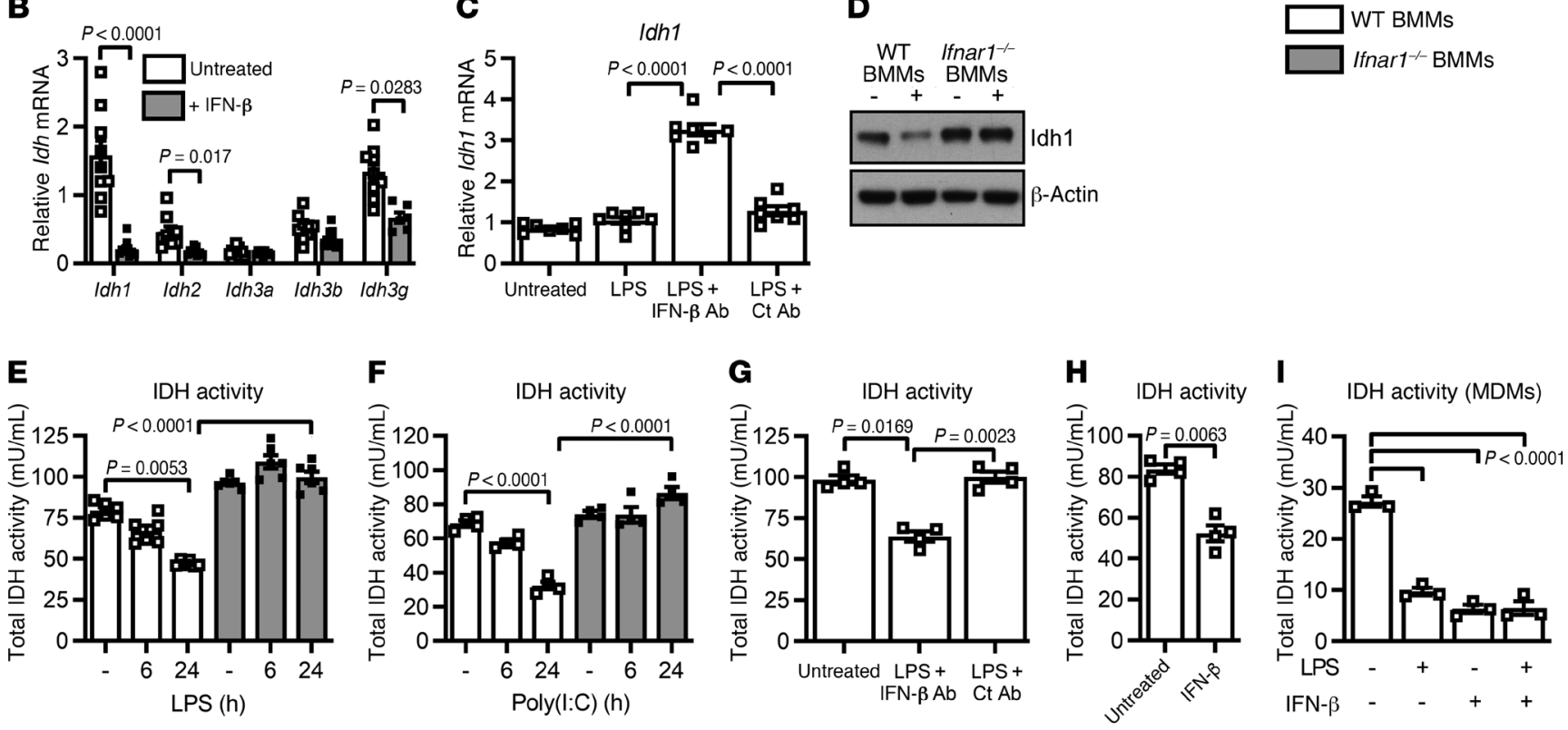

Figure 3. Autocrine type I IFN inhibits IDH in LPS-treated macrophages. Expression of Idh1, Idh2, Idh3a, Idh3b, and Idh3g (A) in WT and Ifnar1 $7^{-/-}$BMMs following LPS (100 ng/mL, 0-24 hours, $n=9$ ) or (B) in BMMs treated with IFN- $\beta$ (1000 U/mL, 24 hours, $n=9)$. (C) Idh1 in BMMs treated with LPS (100 ng/ $\mathrm{mL}, 24$ hours) plus IFN- $\beta$ Ab or Ct Ab $(n=7)$. (D) Idh1 in WT and Ifnar1 ${ }^{-/-}$BMMs following LPS treatment (100 ng/mL, 24 hours). Total IDH activity in WT and Ifnart ${ }^{-1-}$ BMMs following (E) LPS (100 ng/mL, 0-24 hours, $\left.n=6\right)$ or $(\mathbf{F})$ poly(I:C) $(25 \mu \mathrm{g} / \mathrm{mL}, 0-24$ hours, $n=4)$ treatment. Total IDH activity in (G) WT BMMs treated with LPS (100 ng/mL, 0-24 hours, $n=4)$ plus IFN- $\beta$ Ab, Ct Ab, or $(\mathbf{H})$ IFN- $\beta(1000 \mathrm{U} / \mathrm{mL}, 24$ hours, $n=4)$. Total IDH activity in (I) MDMs following LPS (100 ng/mL, 24 hours) with or without IFN- $\beta(1000 \mathrm{U} / \mathrm{mL}, 24$ hours, $n=3)$. Data are mean \pm SEM. $P$ values were determined by 2 -way ANOVA (A, E, and $\mathbf{F})$, 1-way ANOVA (C, G, and I), or Student's $t$ test (B and $\mathbf{H})$.

sion of Idh1 at 6 hours after activation (Figure 3A). In comparison, LPS-stimulated Ifnar1 ${ }^{-/}$BMMs had significantly enhanced gene expression of IdhI (24 hours after LPS treatment) and Idh3a (6 and 24 hours after LPS) compared with untreated Ifnar1 ${ }^{-1}$ BMMs (Figure 3A). The differences in Idh1 expression were the most pronounced, with approximately 5 -fold-higher expression levels in LPS-treated Ifnar1 ${ }^{-1}$ BMMs than in LPS-treated WT BMMs (Figure 3A; 24 hours after LPS). Idh2 (24 hours after LPS) and $I d h 3 a$ ( 6 and 24 hours after LPS) expression levels were also enhanced in LPS activated Ifnar1 $1^{-/}$versus WT BMMs. Further confirming that type I IFN inhibited IDH family gene expression, we found that treatment of WT BMMs with exogenous IFN- $\beta$ led to significantly reduced expression of $I d h 1, I d h 2$, and Idh $3 g$ (Figure 3B) and that neutralization of IFN- $\beta$ in LPS-stimulated WT BMMs significantly increased IdhI expression (Figure 3C). Furthermore, we found that LPS treatment of WT BMMs decreased expression of Idh1, as determined by Western blot analysis, whereas levels were unchanged in LPS-treated Ifnar $1^{-/-}$ BMMs (Figure 3D).
We next treated WT and Ifnar1 ${ }^{-1-}$ BMMs with LPS or poly(I:C) over 24 hours and determined total IDH activity. IDH activity was impaired by approximately $40 \%$ after 24 hours of LPS treatment in WT BMMs (Figure 3E), whereas IDH activity in Ifnar1 $1^{-1-}$ BMMs was unchanged following LPS activation. This distinct response meant that IDH activity in LPS-treated Ifnar1 ${ }^{-/}$BMMs was approximately 2-fold higher than in their WT counterparts. Poly(I:C), a potent inducer of type I IFN (13), blocked IDH activity in WT BMMs at 24 hours (Figure 3F), with no impact observed in Ifnar $1^{-1}$ BMMs. There was a tendency for both LPS and poly(I:C) to reduce IDH activity at 6 hours, but this was not statistically significant. In agreement with the restored carbon flow through IDH in LPS plus anti-IFN- $\beta$ Ab-treated WT BMMs (Figure 2B), we found that IDH enzymatic activity in these cells was restored (Figure 3G). Exogenous IFN- $\beta$ treatment of WT BMMs led to a significant reduction in IDH activity (Figure $3 \mathrm{H}$ ), and LPS-treated human monocyte-derived macrophages (MDMs) also had significantly impaired IDH activity, which could be replicated by exogenous IFN- $\beta$ (Figure 3I). 

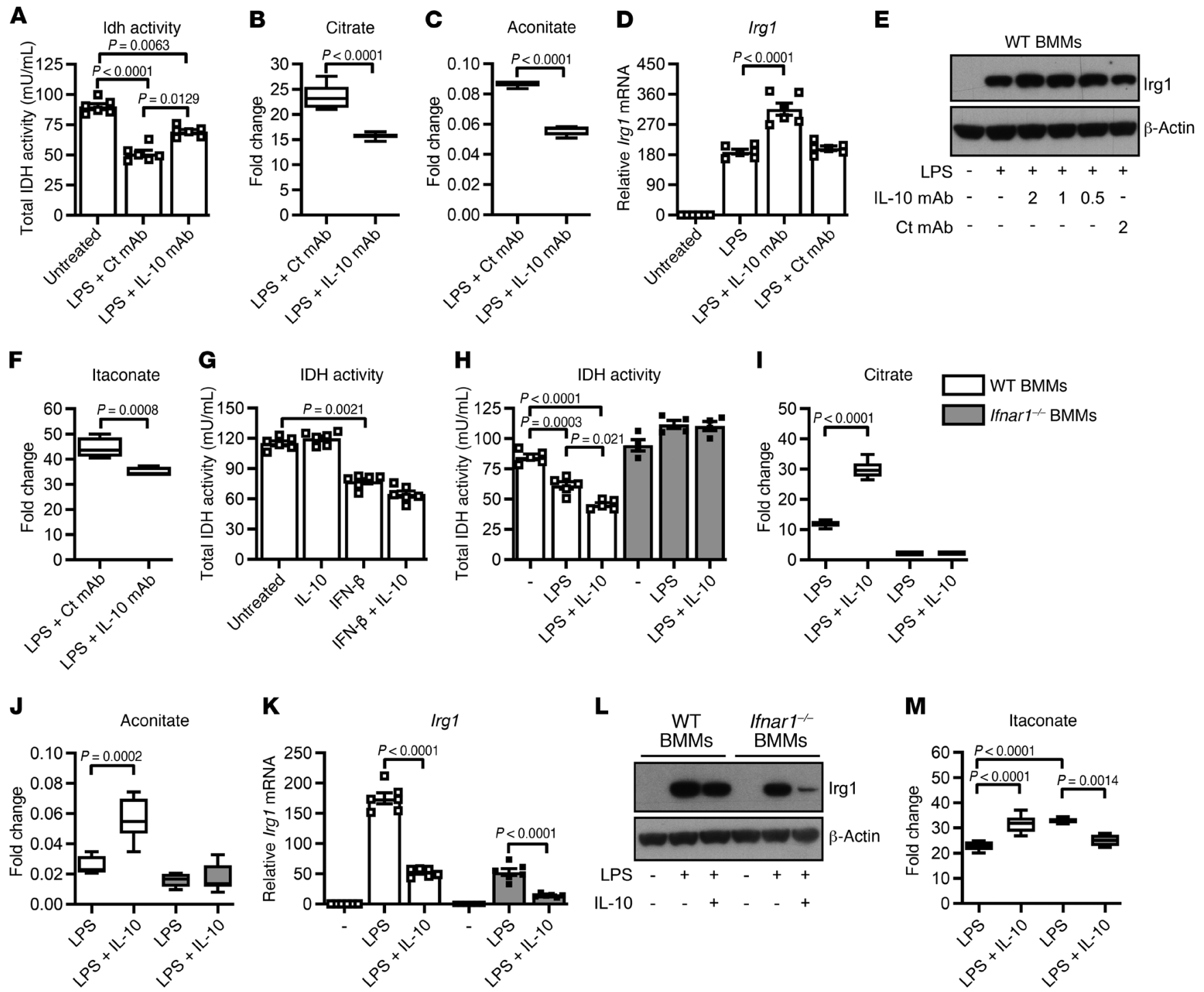

Figure 4. Type I IFN induction of IL-10 contributes to inhibition of IDH. (A) Total IDH activity in WT BMMs treated with LPS (100 ng/mL, $0-24$ hours, $n=6)+$ IL-10 mAb or control mAb $(1 \mu \mathrm{g} / \mathrm{mL})$. (B) Citrate and (C) aconitate in WT BMMs treated as in $\mathbf{A}(n=6)$. (D) Irg1 gene $(n=6)$ and $(\mathbf{E})$ protein in WT BMMs treated with LPS (24 hours) + IL-10 mAb or Ct mAb $(\mu \mathrm{g} / \mathrm{mL})$. (F) Itaconate in WT BMMs treated as in A. (C) Total IDH activity in WT BMMs following IL-10 (100 ng/mL) or IFN- $\beta(1000 \mathrm{U} / \mathrm{mL}, 24$ hours, $n=6)$ treatment. (H) Total IDH activity in WT and Ifnar1--/ BMMs following LPS with or without IL-10 (100 ng/mL, 24 hours, $n=5)$. (I) Citrate and (J) aconitate in WT and Ifnar1 ${ }^{-/-}$BMMs treated as in $\mathbf{H}(n=6)$. (K) Irg1 gene $(n=6)$ and $(\mathbf{L})$ protein in WT and $/ f n a r 1^{-/-}$BMMs treated as in $\mathbf{H}$. (M) Itaconate in WT and Ifnart ${ }^{-1-}$ BMMs treated as in H. Mean \pm SEM. $P$ values by 1-way ANOVA (A, D, G-K, and $\mathbf{M}$ ) or Student's $t$ test (B, C, and F).

We measured the activity of aconitase, which catalyzes the conversion of citrate to isocitrate (1). In agreement with others (14), we found that LPS reduced aconitase activity (Supplemental Figure 1K); however, we found no significant difference in aconitase activity between LPS-treated WT and Ifnar ${ }^{-/}$BMMs. These data provide evidence that autocrine type I IFN is responsible for inhibition of IDH activity in LPS-stimulated macrophages.

Type I IFN induction of IL-1O contributes to inhibition of IDH. Type I IFN drives IL-10 release in macrophages (Supplemental Figure 1L) $(9,15)$, and IL-10 has a profound impact on macrophage metabolism (10). Given this, we first measured IDH activity in LPS-treated WT BMMs in the presence of a neutralizing anti-IL-10 mAb or an isotype control mAb. Blockade of IL-10 partially restored IDH activity in LPS-treated WT BMMs (Figure 4A), although IDH activity was still significantly decreased relative to that in untreated BMMs. Consistent with this finding, accumulation of citrate (Figure 4B) and aconitate (Figure 4C) was reduced in BMMs treated with LPS plus anti-IL-10 mAb relative to LPS plus control mAb-treated BMMs. Blockade of endogenous IL-10 increased Irg1 expression in LPS-treated BMMs (Figure 4, D and E) but, interestingly, led to a reduction in the itaconate pool size in these cells (Figure 4F). Our data agree with an earlier study finding that the first break in the TCA cycle was intact in LPS-treated $\mathrm{Il1O}^{-/-}$BMMs (16). A small flux in metabolite concentration can induce significant changes in enzyme activity (17). The data presented here suggest that blockade of endogenous IL-10 in LPS-stimulated macrophages reduces the accumulation of citrate and aconitate, the substrate for $\operatorname{Irg} 1$ (6), which in turn reduces the generation of itaconate, despite the increased Irg1 expression. 
We next determined the impact of exogenous IL-10 on WT

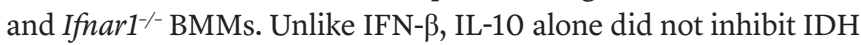
activity in WT BMMs (Figure 4G). However, when combined with IFN- $\beta$, it tended to reduce IDH activity in these cells (Figure $4 G$ ). In combination with LPS, IL-10 led to further inhibition of IDH activity in WT BMMs but had no impact on IDH activity in Ifnar $1^{-1-}$ BMMs (Figure $4 \mathrm{H}$ ). These data suggest that the ability of IL-10 to block IDH activity is dependent on the presence of type I IFN signaling in BMMs. We found by metabolic profiling that, consistent with its blockade of IDH activity in LPS-treated WT BMMs, exogenous IL-10 significantly elevated levels of citrate (Figure 4I) and aconitate (Figure 4J) in these cells. In comparison, IL-10 did not alter the pool sizes of either citrate or aconitate in LPS-treated Ifnar ${ }^{-/-}$BMMs (Figure 4, I and J), in agreement with its inability to block IDH activity in these cells (Figure 4H). Irg1 expression (Figure 4, K and L) was partially blocked by IL-10 in LPS-treated WT BMMs. Blockade of Irg1 protein was more pronounced in LPS-treated Ifnar1 ${ }^{-/-}$BMMs (Figure 4L). IL-10 also blocked IFN- $\beta$ induced Irg1 expression in WT BMMs (Supplemental Figure 1M). IL-10 inhibition of Irg1 expression in macrophages has been shown previously (18). Regarding itaconate levels, we found that IL-10 boosted itaconate synthesis in LPS-treated WT BMMs, whereas IL-10 decreased itaconate in LPS-treated Ifnar1 ${ }^{-/-}$BMMs (Figure $4 \mathrm{M})$. In summary, in LPS-treated WT BMMs, IL-10 blocked IDH activity, resulting in further accumulation of citrate and aconitate to drive itaconate synthesis. In LPS-treated Ifnar1 $1^{-/-}$BMMs, IL-10 had no impact on IDH activity or substrate accumulation, enabling the IL-10-mediated suppression of Irg1, which is more evident in these cells, to impair itaconate synthesis.

We showed that LPS-treated Ifnar1 ${ }^{-/}$BMMs have increased carbon flux, via IDH, toward succinate (Figure 2A) and increased carbon flux, via Irg1, toward itaconate (Supplemental Figure 1I). This distinct carbon flow led to reduced citrate levels in LPS-treated Ifnar1 ${ }^{-/-}$BMMs (Figure 1A), as citrate is utilized to fuel the accumulation of both succinate and itaconate. The elevated flux toward itaconate in LPS-treated Ifnar1 ${ }^{-/-}$BMMs was consistent with their minimal IL-10 production (Supplemental Figure 1L), which uniquely blocked itaconate generation in these cells (Figure 4M). Our results are supported by a detailed analysis of LPS-treated MDMs, where transient induction of $\operatorname{Irg} 1$ and repression of Idh1 mRNA was correlated with the level of induction of IFN- $\beta$ (19). Thus, in the absence of IL-10-mediated suppression of Irg1, carbon flux through Irg1 is increased, leading to the elevated itaconate levels observed in LPS-treated Ifnar1 ${ }^{-/-}$BMMs. Overall, this study demonstrates that inhibition of IDH in the TCA cycle of LPS-stimulated macrophages is due to the autocrine type I IFN pathway.

\section{Methods}

See Supplemental Methods for a detailed description of all experimental procedures.

Study approval. All experiments were performed in accordance with the ethics approval obtained from the University of Melbourne Animal Ethics Committee.

\section{Author contributions}

AJF conceptualized the work. AJF, DPD, and DLT were responsible for methodology. AJF, MKSL, AA, MCL, KJB, and SD validated results. SD, AJM, MJM, KJB, DPD, and DLT were responsible for resources. AJF, MJM, ADC, AJM, and JAH participated in the writing of this article.

\section{Acknowledgments}

This work was supported by National Health and Medical Research Council (NHMRC) grants (APP1107001 and APP1159901). MJM is an NHMRC Principal Research Fellow.

Correspondence: Andrew J. Fleetwood, Department of Medicine, Royal Melbourne Hospital, University of Melbourne, Melbourne, Victoria, Australia. Phone: 61.3.8344.3299; Email: andrew.fleetwood@ unimelb.edu.au.
1. O'Neill LA. A broken Krebs cycle in macrophages. Immunity. 2015;42(3):393-394.

2. Jha AK, et al. Network integration of parallel metabolic and transcriptional data reveals metabolic modules that regulate macrophage polarization. Immunity. 2015;42(3):419-430.

3. Tannahill GM, et al. Succinate is an inflammatory signal that induces IL-1 $\beta$ through HIF- $1 \alpha$. Nature. 2013;496(7444):238-242.

4. Strelko CL, et al. Itaconic acid is a mammalian metabolite induced during macrophage activation. J Am Chem Soc. 2011;133(41):16386-16389.

5. Mills EL, et al. Succinate dehydrogenase supports metabolic repurposing of mitochondria to drive inflammatory macrophages. Cell. 2016;167(2):457-470.e13.

6. Michelucci A, et al. Immune-responsive gene 1 protein links metabolism to immunity by catalyzing itaconic acid production. Proc Natl Acad Sci US A. 2013;110(19):7820-7825.

7. Lampropoulou V, et al. Itaconate links inhibition of succinate dehydrogenase with macrophage metabolic remodeling and regulation of inflammation. Cell Metab. 2016;24(1):158-166.
8. Kretschmer S, Lee-Kirsch MA. Type I interferonmediated autoinflammation and autoimmunity. Curr Opin Immunol. 2017;49:96-102.

9. Fleetwood AJ, Dinh H, Cook AD, Hertzog PJ, Hamilton JA. GM-CSF- and M-CSF-dependent macrophage phenotypes display differential dependence on type I interferon signaling. JLeukoc Biol. 2009;86(2):411-421.

10. Ip WKE, Hoshi N, Shouval DS, Snapper S, Medzhitov R. Anti-inflammatory effect of IL-10 mediated by metabolic reprogramming of macrophages. Science. 2017;356(6337):513-519.

11. Mills EL, et al. Itaconate is an anti-inflammatory metabolite that activates Nrf2 via alkylation of KEAP1. Nature. 2018;556(7699):113-117.

12. Kloosterhof NK, Bralten LB, Dubbink HJ, French PJ, van den Bent MJ. Isocitrate dehydrogenase-1 mutations: a fundamentally new understanding of diffuse glioma? Lancet Oncol. 2011;12(1):83-91.

13. Raj NB, Pitha PM. Analysis of interferon mRNA in human fibroblast cells induced to produce interferon. Proc Natl Acad Sci U S A . 1981;78(12):7426-7430.

14. Samokhvalov V, Jamieson KL, Vriend J, Quan S, Seubert JM. CYP-epoxygenase metabolites of docosahexaenoic acid protect HL-1 cardiac cells against LPS-induced cytotoxicity through SIRT1. Cell Death Discov. 2015;1:15054.

15. Chang EY, Guo B, Doyle SE, Cheng G. Cutting edge: involvement of the type I IFN production and signaling pathway in lipopolysaccharide-induced IL-10 production. JImmunol. 2007;178(11):6705-6709.

16. Baseler WA, et al. Autocrine IL-10 functions as a rheostat for M1 macrophage glycolytic commitment by tuning nitric oxide production. Redox Biol. 2016;10:12-23.

17. Wegner A, Meiser J, Weindl D, Hiller K. How metabolites modulate metabolic flux. Curr Opin Biotechnol. 2015;34:16-22.

18. Gautam A, et al. Interleukin-10 alters effector functions of multiple genes induced by Borrelia burgdorferi in macrophages to regulate Lyme disease inflammation. Infect Immun. 2011;79(12):4876-4892.

19. Baillie JK, et al. Analysis of the human monocyte-derived macrophage transcriptome and response to lipopolysaccharide provides new insights into genetic aetiology of inflammatory bowel disease. PLoS Genet. 2017;13(3):e1006641. 